\title{
Polymer supported nickel complex: Synthesis, structure and catalytic application
}

\author{
ALEKHA KUMAR SUTAR ${ }^{\mathrm{a}, *}$, TUNGABIDYA MAHARANA ${ }^{\mathrm{b}}$, YASOBANTA DAS $^{\mathrm{a}, \mathrm{c}}$ and \\ PRASANTA RATH ${ }^{\mathrm{c}}$ \\ ${ }^{a}$ Catalysis Research Lab, Department of Chemistry, Ravenshaw University, Cuttack - 3, Odisha, India \\ ${ }^{\mathrm{b}}$ Department of Chemistry, National Institute of Technology, Raipur, India \\ ${ }^{\mathrm{c} S}$ School of Applied Sciences (Chemistry), KIIT University, Bhubaneswar, Odisha, India \\ e-mail: dralekhasutar@gmail.com
}

MS received 26 January 2014; revised 8 May 2014; accepted 29 May 2014

\begin{abstract}
In the present investigation, a new synthetic route for a novel recyclable free [3-MOBdMBn-Ni] and polystyrene-anchored [P-3-MOBdMBn-Ni] nickel complexes is presented. The free and polymer-anchored metal complexes were synthesized by the reaction of nickel (II) with one molar equivalent of unsupported $\mathrm{N} \mathrm{N}^{\prime}$-bis (2-Hydroxy-3-methoxybenzaldehyde) 4-Methylbenzene-1,2-diamine (3-MOBdMBn) or polymersupported (P-3-MOBdMBn) Schiff-base ligand in methanol under nitrogen atmosphere. The advantages of these polymer-supported catalysts are the low cost of catalyst and recyclability up to six times, due to easy availability of materials and simple synthetic route. The higher efficiency of complexation of nickel on the polymer-anchored 3-MOBdMBn Schiff base than the unsupported analogue is another advantage of this catalyst system. The structural study reveals that nickel(II) complex of 3-MOBdMBn is square planar in geometry. The catalytic activity of nickel complex towards the oxidation of phenol was investigated in the presence of hydrogen peroxide. Experimental results indicate that the reactivity of P-3-MOBdMBn-Ni was dramatically affected by the polymer support compared to free 3-MOBdMBn-Ni. The rates of oxidation $\left(\mathrm{R}_{\mathrm{p}}\right)$ for unsupported and supported catalysts are $1.37 \times 10^{-6}$ mole $\mathrm{dm}^{-3} \mathrm{~s}^{-1}$ and $2.33 \times 10^{-6}$ mole $\mathrm{dm}^{-3} \mathrm{~s}^{-1}$ respectively.
\end{abstract}

Keywords. Polystyrene support; catalysis; organometallic catalyst, nickel complex; phenol oxidation.

\section{Introduction}

Schiff base catalysts have a great impact on both the scholastic research and polymer industry. Schiff base complexes have shown adaptable applications in different organic transformation reactions such as oxidation, ${ }^{1,2}$ epoxidation of olefins, ${ }^{3,4}$ and polymerization of ethylene. ${ }^{5,6}$ However, it was observed that the homogeneous Schiff-base catalytic systems have two key disadvantages: the lack of control of product which causes the reactor fouling and the limitation of its use in solution processes. Thus, binding these Schiff base metal catalysts onto polymer supports can offer a promising route to prevail over these drawbacks. In general, the heterogeneous Schiff base catalytic system (or supported system) apparently has a lower activity than its corresponding homogeneous analogue. But polymer supported transition metal complexes have shown high catalytic activity ${ }^{7,8}$ in comparison to homogeneous and unsupported catalysts. Also, polymer-supported catalysts are easily recovered without any substantial loss

*For correspondence in their catalytic activity ${ }^{9,10}$ but homogeneous catalysts are not recovered easily.

Further, oxidation of phenol is an industrially important reaction as its products catechol and hydroquinone are widely used as antioxidants, polymerization inhibitors, photography chemicals, flavouring agents, drug intermediates, etc. Since 1970, phenol oxidation, has been widely investigated using various homogeneous and heterogeneous catalysts. ${ }^{11}$ Moreover, phenol is an intermediate in the oxidation of many aromatic compounds and a toxic molecule resistant to biotreatment. For the treatment of wastewater containing highly concentrated, toxic or hardly biodegradable compounds, oxidation of such organic pollutants, into non-toxic products in the presence of a catalyst is a promising approach. ${ }^{12,13}$

Oxidation of phenols using various chemical reagents such as, hydrogen peroxide, permanganate, molecular oxygen and ozone, are extensively used. ${ }^{14}$ The use of hydrogen peroxide has the benefit of producing oxygen which can be used to supplement biological degradation. ${ }^{15}$ Oxidation of phenol using unsupported Schiff base complexes of metal ions is reported, ${ }^{16}$ but 
the oxidation of phenol using polymer-supported transition metal complexes was found to be high ${ }^{17}$ in the presence of tert-butyl hydroperoxide (t-BHP). The activity of polymer-supported Schiff base complexes of iron(III), cobalt(II) and nickel(II) ions in the oxidation of phenol ${ }^{18,19}$ also showed variation with temperature, time, etc, which might be due to the change in concentration of substrate or catalyst.

Although the oxidation of phenol in the presence of metal complexes of salen and hydrogen peroxide as the oxidant is reported earlier ${ }^{8}$ the catalytic activity of metal complexes of N, N'-bis (2-hydroxy-3methoxybenzaldehyde) 4-Methylbenzene-1,2-diamine Schiff base (3-MOBdMBn) is not reported in the literature. Hence, in the present investigation an attempt has been made to prepare polymer-supported nickel complex of 3-MOBdMBn Schiff base and to characterize them for their structures and catalytic activity towards the oxidation of phenol in the presence of hydrogen peroxide as an oxidant. Here the substituent 4-methyl and 3-methoxy play a significant role towards the stability as well as in the catalytic activity of the $\mathrm{Ni}$ (II) complex.

\section{Experimental}

\subsection{Materials}

Divinyl benzene cross-linked chloromethylated polystyrene beads were obtained from Ion Exchange India Ltd., Mumbai, India and were used to anchor synthesized 3-MOBdMBn Schiff base complexes of metal ions. The anhydrous chloro salts of nickel(II) ions were purchased from Ranbaxy, Mumbai, India and used without further purifications. The phenol, hydrogen peroxide (30.0 wt\%), 2-hydroxy-3-methoxybenzaldehyde (3-MOBd) and 4-Methylbenzene-1,2-diamine (MBn) were procured form E. Merck, India. Other chemicals and solvents were of analytical grade $(>99.0 \mathrm{wt} \%)$ and used after drying with standard methods. ${ }^{20}$

\subsection{Characterization of 3-MOBdMBn Schiff base and its metal complexes}

IR spectra of 3-MOBdMBn Schiff base and its nickel complexes were recorded on $\mathrm{KBr}$ pellet using PerkinElmer 1600 FTIR Spectrophotometer. The electronic spectra of 3-MOBdMBn Schiff base and its nickel complexes were recorded with Shimadzu 1601 PC UV-Vis Spectrophotometer by using sample mull in a cuvette. Thermogravimetric analysis (TGA) of 3MOBdMBn Schiff base and its nickel complexes were carried out using Perkin-Elmer Pyris, Diamond Thermal Analyzer under nitrogen atmosphere at a heating rate of $5^{\circ} \mathrm{C} / \mathrm{min}$. The loading of metal ions on 3MOBdMBn Schiff base was determined by analyzing the solution with PerkinElmer 3100 Atomic Absorption Spectrometer at $\lambda_{\max }$ of nickel ion. The amount of 3MOBdMBn Schiff base anchored on polymer beads and its composition were estimated using Haraeus Carlo Ebra 1108 Elemental Analyzer. The ${ }^{1} \mathrm{H}-\mathrm{NMR}$ spectra of 3-MOBdMBn Schiff base and its nickel complexes were recorded on an FT-NMR-Brucker $300 \mathrm{MHz}$ Spectrometer using DMSO- $d^{6}$ as a solvent and tetramethylsilane (TMS) as an internal reference. The magnetic moment $(\mu)$ of nickel complexes were measured using Vibrating Sample Magnetometer-155. The molecular weight of 3-MOBdMBn Schiff base and its nickel complexes were determined using a Vapor Pressure Osmometer (Merk VAPRO 5600, Germany).

\subsection{Synthesis of 3-MOBdMBn Schiff base and its nickel complex}

The 3-MOBdMBn Schiff base was synthesized by the modified procedure reported in the literature. ${ }^{7}$ The reaction mixture containing 2-hydroxy-3methoxybenzaldehyde $(20 \mathrm{mmol}, 3.043 \mathrm{~g})$ and 4Methylbenzene-1,2-diamine (10 mmol, $1.22 \mathrm{~g})$ in methanol was refluxed at $60^{\circ} \mathrm{C}$ for about $1 \mathrm{~h}$. On cooling at low temperature the reaction mixture produced light orange red coloured crystals, which were filtered and recrystallized with methanol. The metal complexes of 3-MOBdMBn Schiff base were prepared by taking $100 \mathrm{~mL}$ methanolic solution of mixture of Schiff base $(20 \mathrm{mmol}, 7.81 \mathrm{~g})$ and $20 \mathrm{mmol}$ of Nickel metal ions in a round bottom flask and refluxing at $60^{\circ} \mathrm{C}$. After $8 \mathrm{~h}$, the solution was cooled and crystalline metal complexes were separated from the mother liquor. Finally, metal complexes were recrytallized in methanol and dried in a vacuum desiccator.

\subsection{Synthesis of polymer-anchored 3-MOBdMBn Schiff base and its nickel complex}

To prepare polymeranchored nickel complexes of 3-MOBdMBn Schiff base, the N, N'-bis (4-amino-2hydroxy-3-methoxybenzaldehyde) 4-Methylbenzene1,2-diamine (A-3-MOBdMBn) Schiff base was prepared by carrying out nitrosation and reduction reactions of 3-MOBdMBn Schiff base and then reacting resulted A-3-MOBdMBn Schiff base with cross-linked chloromethylated polystyrene beads. The nitrosation of 3-MOBdMBn Schiff base was carried 
out using $20 \mathrm{mmol}$ (7.81 g) of 3-MOBdMBn Schiff base with sodium nitrite $(20 \mathrm{mmol})$ in $1.0 \mathrm{~N}$ hydrochloric acid $(100 \mathrm{~mL})$ in an ice bath. The resultant $\mathrm{N}$, N'-bis (4-nitroso-2-hydroxy-3-methoxybenzaldehyde) 4-Methylbenzene-1,2-diamine (NO-3-MOBdMBn) was filtered and washed with hot and cold water to remove reaction impurities. The reduction of NO$3-M O B d M B n$ was carried out using $20 \mathrm{mmol}$ of nitrosated Schiff base in $1.0 \mathrm{~N}$ hydrochloric acid $(50 \mathrm{~mL})$ in the presence of metallic iron, which produced A-3-MOBdMBn Schiff base. To immobilize the prepared A-3-MOBdMBn Schiff base on cross-linked chloromethylated polystyrene, the methanol swollen polymer beads $(5 \mathrm{~g})$ were refluxed in $50 \mathrm{~mL}$ methanol containing $20 \mathrm{mmol}$ (8.41 g) A-3-MOBdMBn Schiff base. After $8 \mathrm{~h}$, the 3-MOBdMBn Schiff base anchored polymer beads were separated and dried in a vacuum desiccator. The amount of 3-MOBdMBn Schiff base loaded on polymer beads was estimated with elemental analysis. Subsequently, the nickel ion was loaded by keeping 3-MOBdMBn loaded polymer beads (5 g) for $10 \mathrm{~h}$ in an aqueous solution $(50 \mathrm{~mL})$ of nickel ion (20 mmol). Finally, the polymer beads were separated and dried at $70^{\circ} \mathrm{C}$ in a vacuum oven after washing with hot and cold water. The loading of nickel ion on polymer bead was determined by analysing the solution with an Atomic Absorption Spectrometer. The loading of nickel ion on free and polymer-supported 3-MOBdMBn Schiff base was calculated as complexation of nickel ion using the amount of 3-MOBdMBn Schiff base taken initially and the amount of nickel ion loaded on polymer beads.

Complexation of Nickel ion

$$
=\frac{\text { Amount of Nickel loaded }}{\text { Amount of Schiff base used for loading }} \times
$$

\subsection{Catalytic activity of metal complexes towards oxidation of phenol}

To evaluate the catalytic activity of free and polymersupported nickel complexes of 3-MOBdMBn Schiff base, the oxidation of phenol was carried out using hydrogen peroxide as the oxidant at fixed ionic strength $(0.10 \mathrm{M})$ and hydrogen ions concentration $(\mathrm{pH} 7.0)$ in the reaction mixture. To carry out these reactions, a calculated amount of metal-anchored polymer beads were taken in a two-necked roundbottomed flask containing $0.05 \mathrm{M}$ phenol $(4.7 \mathrm{~g})$. The oxidation of phenol was carried out adding $5.67 \mathrm{~g}(0.05 \mathrm{M})$ hydrogen peroxide $(30 \mathrm{wt} \%)$ in the reaction mixture and $2 \mathrm{~mL}$ chlorobenzene as an internal standard. The water condenser and supply of nitrogen were attached with reaction flask before starting the heating and stirring (1200 rpm) of reaction mixture. Gas chromatography was used to follow these reactions at different time intervals. The retention time of standards was used to identify the reaction products, and product selectivity was measured using peak areas of reaction products in the chromatograms. The reactions were also carried out taking different molar ratios of substrates, hydrogen peroxide, and catalyst. The rate of oxidation for the oxidation of phenol was determined by studying reactions at different temperatures at constant molar ratios of substrate to $\mathrm{H}_{2} \mathrm{O}_{2}$ and catalyst. The reactions were also studied without using catalysts to compare the effect of catalyst towards the oxidation of phenol.

\section{Results and Discussion}

Investigations have revealed that polymer-supported metal complexes are sometimes more catalytic and efficient than free and unsupported analogues. ${ }^{18,19}$ The activity of catalysts also showed dependence on properties of polymer supports and amount of loaded catalysts. Thermal stability of catalysts is required to be high, if these catalysts have to be applied for high temperature reactions. Therefore, thermal analysis of supported metal complexes was carried out to evaluate their possible applications in high temperature reactions and to provide a proof for complexation of metal ions with polymer-anchored 3-MOBdMBn Schiff base. The TGA of polymer supported 3-MOBdMBn Schiff base (figure 1) showed a weight loss of $39.1 \mathrm{wt} \%$ at $500^{\circ} \mathrm{C}$, but its nickel(II) ion complex showed a weight loss of $35.1 \mathrm{wt} \%$ at the same temperature. This was a clear indication that nickel(II) ion complex was more stable than its Schiff base ligand. ${ }^{21}$ Also, the nickel complex of P- 3MOBdMBn Schiff base was more stable than the polymer supported nickel complex synthesized by Gupta et al. ${ }^{19}$ (showed a weight loss of $45 \mathrm{wt} \%$ at $500^{\circ} \mathrm{C}$ ), may be due to the presence of 4-methyl and 3-methoxy substituent in the Schiff base.

Further, polymer-supported 3-MOBdMBn Schiff base and its nickel complex were also characterized by IR and UV techniques to provide a proof for the complexation of metal ions and to decide the structures and geometry of the complex on the basis of elemental analysis and magnetic properties.

\subsection{Characterization of 3-MOBdMBn Schiff base}

The yield of 3-MOBdMBn Schiff base was found to be $91.6 \mathrm{wt} \%$ (scheme 1). The IR spectrum of 3MOBdMBn Schiff base showed absorption bands at 


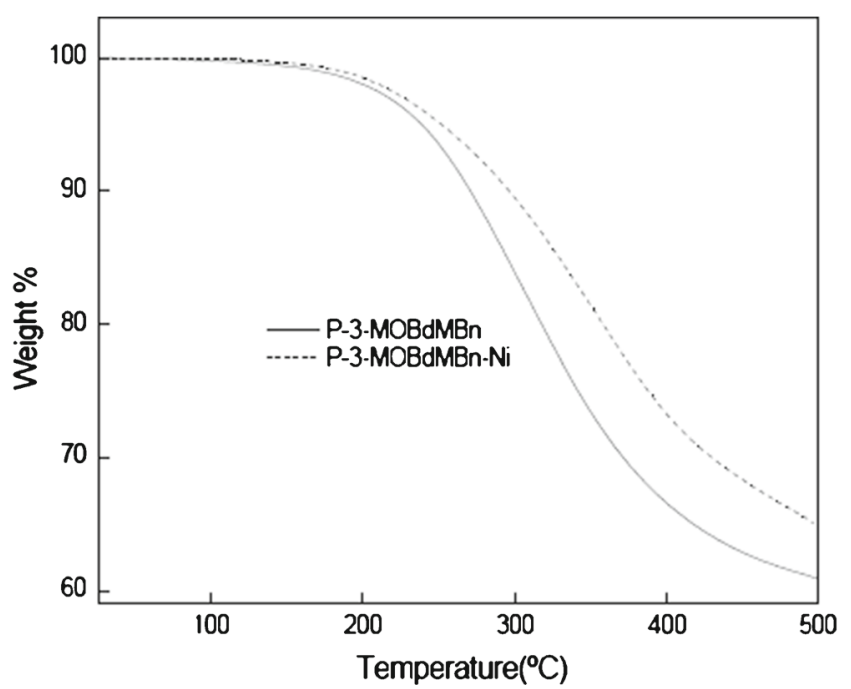

Figure 1. Thermal stability of polymer-supported 3MOBdMBn Schiff base and its nickel complex.

$1609 \mathrm{~cm}^{1}(>\mathrm{C}=\mathrm{N}), 1263 \mathrm{~cm}^{1}(>\mathrm{C}-\mathrm{O})$ phenolic $(\mathrm{SI})$. The elemental analysis of 3-MOBdMBn Schiff base proved (wt \%): $\mathrm{C}=69.82, \mathrm{~N}=7.03$ and $\mathrm{H}=6.31$; Caltd (\%): $\mathrm{C}=70.75, \mathrm{~N}=7.17$ and $\mathrm{H}=5.68$, which corresponds to $\mathrm{C}_{23} \mathrm{H}_{22} \mathrm{~N}_{2} \mathrm{O}_{4}$ empirical formula of 3-MOBdMBn Schiff base. ${ }^{7,22}$

The molecular weight of Schiff base was $389.37 \mathrm{~g} \mathrm{~mol}^{-1}$ (Caltd $390.43 \mathrm{~g} \mathrm{~mol}^{-1}$ ). The electronic spectra of 3-MOBdMBn Schiff base showed absorption bands at $289 \mathrm{~nm}$ and $347 \mathrm{~nm}$, which were assigned to $\pi \rightarrow \pi^{*}$ and $\mathrm{n} \rightarrow \pi^{*}$ transitions. The ${ }^{1} \mathrm{H}-\mathrm{NMR}$ spectrum of 3-MOBdMBn Schiff base (figure S3) showed signals at $\delta / \mathrm{ppm}=2.79(3 \mathrm{H}), 3.81(6 \mathrm{H}), 5.13(2 \mathrm{H})$, $6.80(2 \mathrm{H}), 6.98(3 \mathrm{H}), 7.25(2 \mathrm{H}), 7.41(2 \mathrm{H})$ and $8.61(2 \mathrm{H})$ which corresponded to the structure of 3-MOBdMBn Schiff base as shown in scheme 1 .

\subsection{Synthesis and characterization of A-3-MOBdMBn Schiff base and its anchoring on polymer beads}

The nitrosation of 3-MOBdMBn Schiff base produced $87.5 \%$ yield of NO-3-MOBdMBn (scheme 2).
It is observed from the elemental analysis of NO-3MOBdMBn that (wt\%): $\mathrm{C}=62.01, \mathrm{~N}=12.17$, and $\mathrm{H}=4.95$; Caltd (wt\%): $\mathrm{C}=61.60, \mathrm{~N}=12.49$ and $\mathrm{H}=4.50$, which corresponds to $\mathrm{C}_{23} \mathrm{H}_{20} \mathrm{~N}_{4} \mathrm{O}_{6}$ formula of nitrosated Schiff base. The molecular weight of NO-3MOBdMBn was $447.02 \mathrm{~g} \mathrm{~mol}^{1}$ (Caltd $448.43 \mathrm{~g} \mathrm{~mol}^{1}$ ). The IR spectrum of NO-3-MOBdMBn showed absorption bands at $1604 \mathrm{~cm}^{1}(>\mathrm{C}=\mathrm{N}), 1260 \mathrm{~cm}^{-1}(>\mathrm{C}-\mathrm{O})$ phenolic, and $1533 \mathrm{~cm}^{-1}$ and $131 \mathrm{~cm}^{-1}$ for $\mathrm{N}-\mathrm{O}$ group. The NO-3-MOBdMBn Schiff base showed a shift in NMR signals in comparison to NMR signals observed for 3-MOBdMBn Schiff base. The NO3-MOBdMBn Schiff base showed proton signals at $\delta / \mathrm{ppm}=2.82(3 \mathrm{H}), 3.75(6 \mathrm{H}), 5.15(2 \mathrm{H}), 7.15(2 \mathrm{H})$, $7.36(3 \mathrm{H}), 7.66(2 \mathrm{H})$ and $8.64(2 \mathrm{H})$, which corresponded to the structure of nitrosated 3-MOBdMBn Schiff base as shown in scheme 2. The protons ortho to nitroso group in 3-MOBdMBn Schiff base were deshielded. Hence, their signals appeared at 7.15 and $7.66 \mathrm{ppm}$ instead of 6.8 and $7.41 \mathrm{ppm}$ respectively, as observed for pure 3-MOBdMBn Schiff base. The proton signal at $7.25 \mathrm{ppm}$ was missing due to the substitution of nitroso group in the benzene ring. The reduction of NO-3-MOBdMBn produced $81.3 \mathrm{wt} \%$ yield of A3MOBdMBn Schiff base as shown in scheme 2. The A3MOBdMBn Schiff base was characterized by different techniques. The elemental analysis of A3-MOBdMBn Schiff base showed (wt $\%$ ): $\mathrm{C}=63.92 \mathrm{~N}=13.21$ and $\mathrm{H}=7.02$, Caltd (wt\%): $\mathrm{C}=65.7 \mathrm{~N}=13.33$, and $\mathrm{H}$ $=5.75$, which corresponded to $\mathrm{C}_{23} \mathrm{H}_{24} \mathrm{~N}_{4} \mathrm{O}_{4}$ empirical formula of Schiff base. The molecular weight of A-3MOBdMBn Schiff base was found to be $418.23 \mathrm{~g} \mathrm{~mol}^{1}$ (Caltd $420.46 \mathrm{~g} \mathrm{~mol}^{1}$ ). The IR spectrum of A-3MOBdMBn Schiff base showed absorption bands at $1600 \mathrm{~cm}^{1}(>\mathrm{C}=\mathrm{N}), 1255 \mathrm{~cm}^{1}(>\mathrm{C}-\mathrm{O})$ phenolic, and a band between 1641 and $1619 \mathrm{~cm}^{1}$ for $>\mathrm{C}-\mathrm{N}$ group. The ${ }^{1} \mathrm{H}-\mathrm{NMR}$ spectrum of A-3-MHBdMBn Schiff base showed proton signals at $\delta / \mathrm{ppm}=2.81(3 \mathrm{H}), 3.72(6 \mathrm{H})$, $4.15(4 \mathrm{H}), 5.15(2 \mathrm{H}), 6.16(2 \mathrm{H}), 6.46(2 \mathrm{H}), 7.36(3 \mathrm{H})$ and $8.63(2 \mathrm{H})$, which corresponded to the structure of A-3MOBdMBn Schiff base as shown in scheme 2.

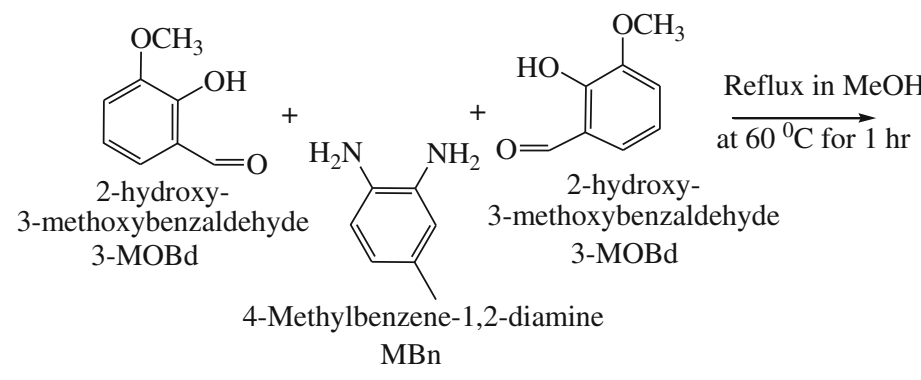

Scheme 1. Synthesis of 3-MOBdMBn Schiff base.

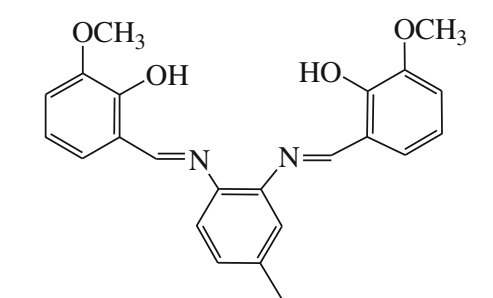

$\mathrm{N}, \mathrm{N}$-bis (2-hydroxy-3-methoxybenzaldehyde 4-Methylbenzene-1,2-diamine Schiff base 3-MOBdMBn 
<smiles>COc1cccc(/C=N/c2ccc(C)cc2/N=C/c2cccc(OC)c2O)c1O</smiles>

3-MOBdMBn

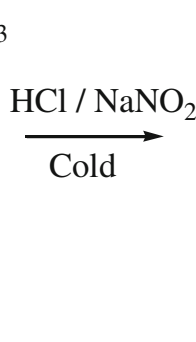<smiles>COc1cc([N+](=O)[O-])cc(/C=N/c2ccc(C)cc2)c1O</smiles>

NO- 3-MOBdMBn<smiles>COc1cc(N=O)cc(C#N)c1O</smiles>

$\mathrm{Fe} / \mathrm{HCl}$<smiles>COc1cc(N)cc(/C=N/c2ccc(C)cc2/N=C/c2cc(N)cc(OC)c2O)c1O</smiles>

A - 3-MOBdMBn

Scheme 2. Synthesis of A-3-MOBdMBn Schiff base.

The synthesized A-3-MOBdMBn Schiff base was anchored on cross-linked chloromethylated polystyrene beads by refluxing A-3-MOBdMBn Schiff base with polymer beads in $\mathrm{DMF}$ for $8 \mathrm{~h}$ at $60^{\circ} \mathrm{C}$. The amount of A-3-MOBdMBn Schiff base anchored on polymer beads was $881 \mathrm{wt} \%$ (scheme 3). The anchoring of A3-MOBdMBn Schiff base on polymer beads was confirmed by comparing the IR spectrum of 3-MOBdMBn Schiff base anchored polymer beads with IR spectrum of pure polymer beads. The IR spectrum of polymeranchored Schiff base showed new absorption bands at $1594 \mathrm{~cm}^{-1}(>\mathrm{C}=\mathrm{N}), 1246 \mathrm{~cm}^{-1}(>\mathrm{C}-\mathrm{O})$ phenolic, and a broad band between $1644 \mathrm{~cm}^{-1}(>\mathrm{C}=\mathrm{N})$, which were absent in the IR spectrum of pure polymer beads, but were present in free Schiff base. The IR spectrum of pure polymer beads showed absorption band at $1262 \mathrm{~cm}^{-1}$, which is attributed to $\mathrm{C}-\mathrm{Cl}$ bond of chloromethyl in cross-linked polymer beads. ${ }^{7}$ The decrease in the intensity of absorption band at $1262 \mathrm{~cm}^{1}$ in polymer- anchored 3-MOBdMBn Schiff base than pure polymer beads was an evidence for anchoring of 3-MOBdMBn Schiff base on polymer beads. The appearance of new absorption bands and shift in characteristic absorption bands of 3-MOBdMBn Schiff base were also used as evidence for anchoring of 3MOBdMBn Schiff base on polymer beads.

\subsection{Characterization of free and polymer-anchored metal complexes of 3-MOBdMBn Schiff base}

The loading of nickel ion on free and polymersupported 3-MOBdMBn Schiff base was carried out by refluxing free Schiff base (scheme 4) and polymeranchored Schiff base in solution of metal salt at $60^{\circ} \mathrm{C}$ for $6 \mathrm{~h}$ (scheme 5). The metal complexes of free Schiff base (3-MOBdMBn-Ni) and polymer-anchored Schiff base (P-3-MOBdMBn -Ni) after separation and

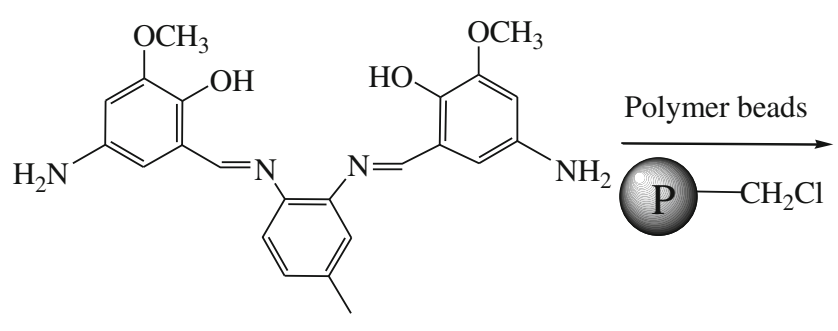

A - 3-MOBdMBn

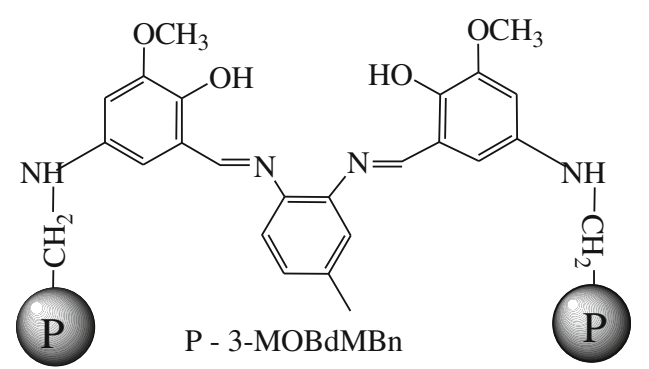

Scheme 3. Synthesis of polymer-anchored Schiff base (P-3-MOBdMBn). 
<smiles>COc1cccc(/C=N/c2ccc(C)cc2/N=C/c2cccc(OC)c2O)c1O</smiles>

3-MOBdMBn
Nickel salt solution

reflux, rt<smiles></smiles>

$\mathrm{Ni}$ - 3-MOBdMBn

Scheme 4. Loading of nickel ion on unsupported 3-MOBdMBn Schiff base.

purification were analyzed for their structures and loading of nickel ion. The complexation of nickel(II) ion on free 3-MOBdMBn Schiff base and polymeranchored Schiff base was 86.34 and 89.88 wt\% respectively (table 1). These results have clearly suggested that the loading of nickel ion on polymersupported 3-MOBdMBn Schiff base was higher than free 3MOBdMBn Schiff base.

The complexation of nickel ion with 3-MOBdMBn Schiff base showed significant variations in IR bands for $>\mathrm{C}=\mathrm{N}$ and $>\mathrm{C}-\mathrm{O}$ groups and new absorption bands appeared due to the formation of $\mathrm{M}-\mathrm{O}$ and $\mathrm{M}-\mathrm{N}$ bonds in metal complex. The disappearance of phenolic broad band in the IR spectrum of 3-MOBdMBn Schiff base after the complexation of nickel ion was an evidence for the complexation of metal ions with 3-MOBdMBn Schiff base. The polymer-supported Schiff base showed absorption bands at low frequency in comparison to free Schiff base (SI) On complexation with nickel(II) ion, the frequency of $>\mathrm{C}=\mathrm{N}$ absorption band of free Schiff base has decreased from 1609 to $1595 \mathrm{~cm}^{-1}$, whereas polymer- supported Schiff base showed variation from 1594 to $1582 \mathrm{~cm}^{-1}$ (figure 2). ${ }^{7}$

These variations in IR band corresponding to $>\mathrm{C}=\mathrm{N}$ group of 3-MOBdMBn Schiff base were used as evidence for interaction of metal ions with azomethine nitrogen $(>\mathrm{C}=\mathrm{N}$ ) of 3-MOBdMBn Schiff base. The complexation of nickel(II) ions showed new absorption band at $424 \mathrm{~cm}^{-1}$ with free Schiff base<smiles>COc1cc(NCc2ccccc2)cc(/C=N/c2ccc(C)cc2/N=C/c2cc(NCc3ccccc3)cc(OC)c2O)c1O</smiles>

P- 3-MOBdMBn

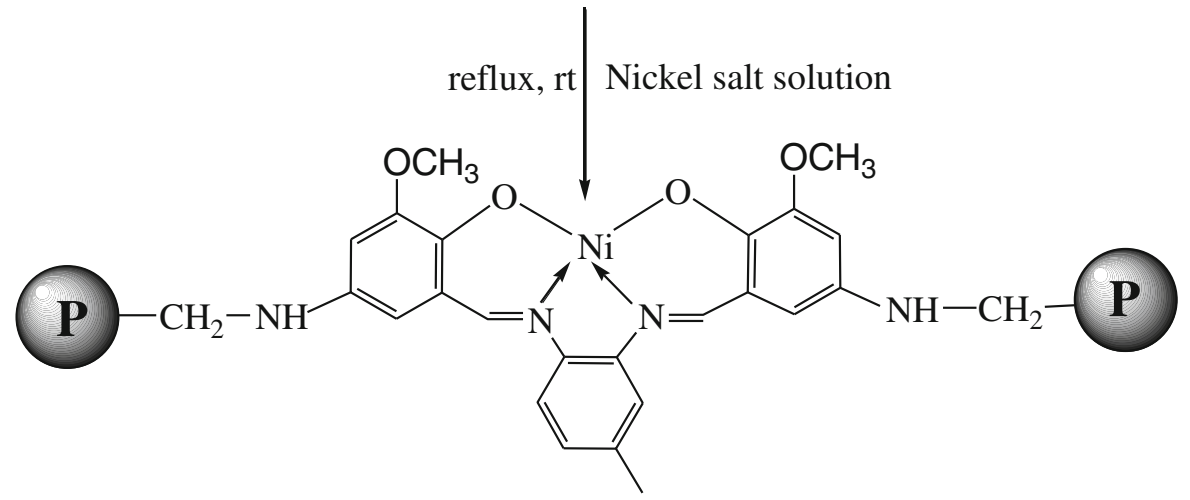

P- 3-MOBdMBn - Ni

Scheme 5. Loading of nickel ion on polymer-supported Schiff base (P-3-MOBdMBn-Ni). 
Table 1. Efficiency of complexation (EC) of nickel ion (wt\%).

\begin{tabular}{lc}
\hline Schiff base & $\begin{array}{c}\text { Complexation (EC) } \\
\text { of nickel(II) ion (wt } \%)\end{array}$ \\
\hline 3-MOBdMBn & 86.34 \\
P-3-MOBdMBn & 89.88 \\
\hline
\end{tabular}

$[3-\mathrm{MOBdMBn}]=20 \mathrm{mmol}$, [Nickel ion $]=20 \mathrm{mmol}$.

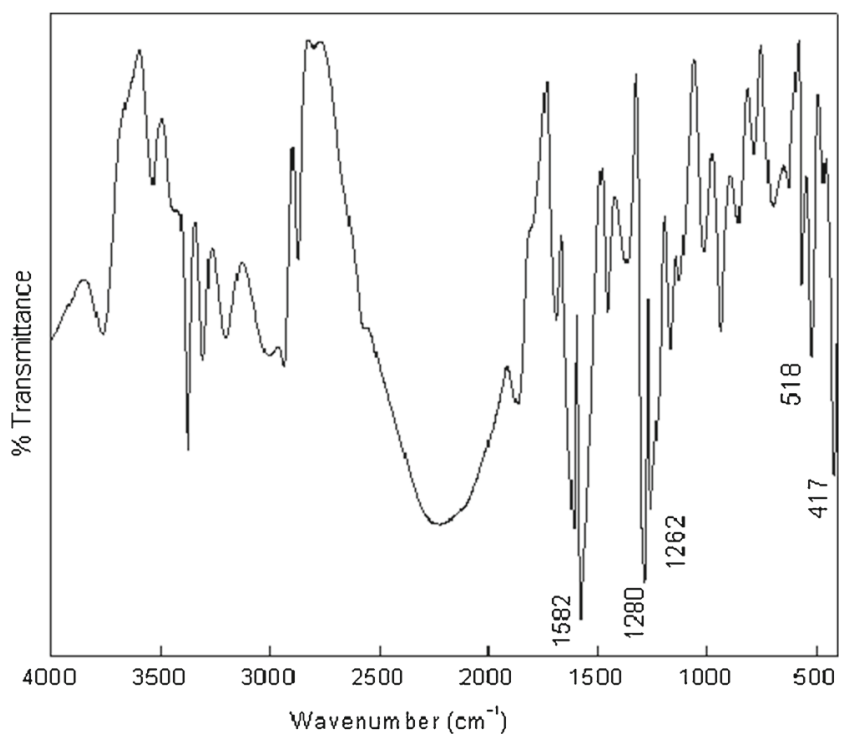

Figure 2. FTIR spectrum of polymer supported 3-MOBdDBn-Ni complex.

and at $417 \mathrm{~cm}^{-1}$ with polymer-anchored Schiff base, which was due to the formation of $\mathrm{M}-\mathrm{N}$ bond between nickel(II) ion and Schiff base. The complexation of nickel(II) ion showed another new band due to the formation of bond between nickel and phenolic oxygen $(-\mathrm{O}-\mathrm{M})$ with polymer-supported 3-MOBdMBn Schiff base at $518 \mathrm{~cm}^{-1}$ (figure 2).

The complexation of metal ions with Schiff base was further confirmed by comparing electronic spectra of nickel complex and pure 3-MOBdMBn Schiff base. The complexation of nickel(II) ion with 3-MOBdMBn Schiff base showed variation in $\pi \rightarrow \pi^{*}$ transition from $289 \mathrm{~nm}$ to $280 \mathrm{~nm}$ and (table 2) the $\mathrm{n} \rightarrow \pi^{*}$ transition of 3-MOBdMBn Schiff base was changed from $347 \mathrm{~nm}$ to $299 \mathrm{~nm}$. The charge transfer (CT) and $\mathrm{d} \rightarrow \mathrm{d}$ transitions were also used as evidence for complexation of nickel with Schiff base. These electronic transitions corresponded to $t_{2 g}^{6} e_{g}^{2}$ configurations for nickel(II) ion in this complex. The magnetic moment $(\mu)$ of nickel Schiff base complex was found to be $0.83 \mathrm{BM}$, which indicated that nickel(II) ion complex was diamagnetic in nature with zero unpaired electron. The magnetic
Table 2. Electronic transitions in unsupported and polymer supported nickel complex of 3-MOBdMBn Schiff base.

\begin{tabular}{lcccc}
\hline & \multicolumn{4}{c}{ Frequencies $\lambda_{\max } / \mathrm{nm}$} \\
\cline { 2 - 5 } Compounds & $\pi \rightarrow \pi *$ & $\mathrm{n} \rightarrow \pi *$ & $\mathrm{C} \rightarrow \mathrm{T}$ & $\mathrm{d} \rightarrow \mathrm{d}$ \\
\hline 3-MOBdMBn & 289 & 347 & - & - \\
P-3-MOBdMBn & 282 & 344 & - & - \\
3-MOBdMBn -Ni & 280 & 299 & 315 & 403 \\
P-3-MOBdMBn -Ni & 273 & 291 & 308 & 398 \\
\hline
\end{tabular}

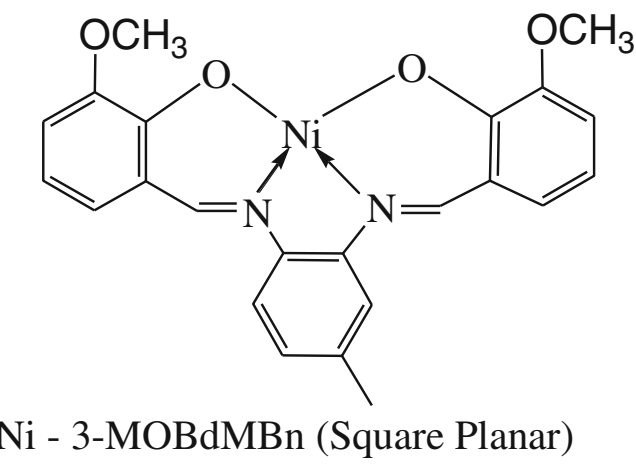

Scheme 6. Geometry and structure of 3-MOBdMBn Schiff base complex of nickel ion.

moment $(\mu)$ and electronic configurations have suggested a square planar structure with $\mathrm{dsp}^{2}$ hybridization for nickel complex (scheme 6).

\subsection{Oxidation of phenol}

The catalytic activity of free and polymer-supported nickel complex of 3-MOBdMBn Schiff base was evaluated by studying the oxidation of phenol in the presence of hydrogen peroxide. The gas chromatographic analysis was used to determine the product selectivity and to estimate the percent conversion of phenol. The catechol (CTL) was a major reaction product in the oxidation of phenol (scheme 7). The formation of reaction products was attributed to enzymatic behaviour of metal complexes of 3-MOBdMBn Schiff base.

The polymer support has facilitated the decomposition of the intermediates. Hence, $\%$ conversion of phenol was more with polymer-supported nickel complex (figure 3) in comparison to free complex of 3MOBdMBn Schiff base (SI). The conversion of phenol increased up to $240 \mathrm{~min}$ and after that the conversion became almost constant due to substantial decrease in the concentration of oxidant and substrate in the reaction mixture for both supported and unsupported catalysts (figure 3 ). The oxidation reaction also had shown 


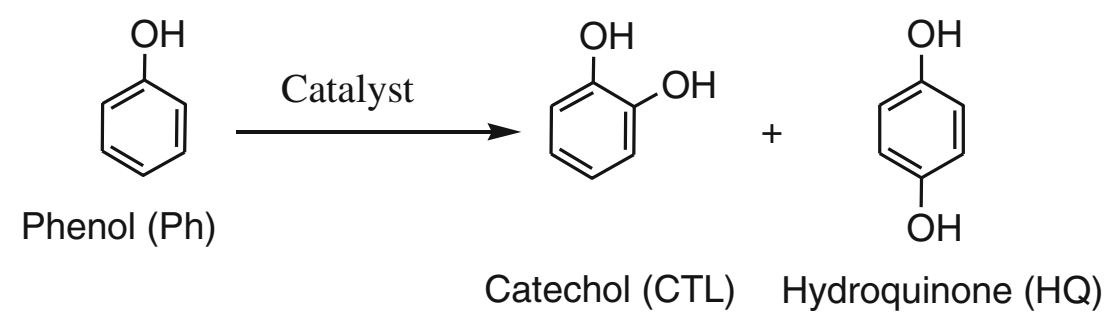

Scheme 7. Oxidation of phenol.

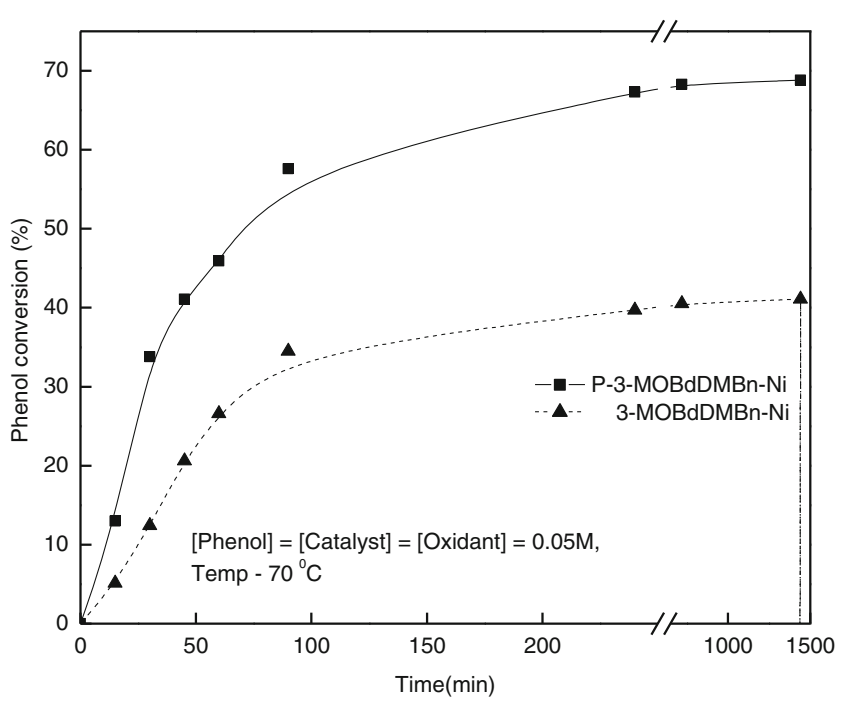

Figure 3. Effect of reaction time on oxidation of phenol in presence of polymer-supported and unsupported nickel complexes. [Phenol]:[Catalyst]: $\left[\mathrm{H}_{2} \mathrm{O}_{2}\right]=1: 1: 1(0.05 \mathrm{M})$, Temp. $=70^{\circ} \mathrm{C}$.

dependence on the type of catalyst. The high activity of polymer supported metal complexes was due to more facile interactions of the catalyst with substrate than with unsupported catalysts. The low activity of catalyst in solution was due to the formation of inactive dimers or multimers of metal complexes in the solution. The amount of phenol oxidized with hydrogen peroxide was almost equal to the sum of amount of CTL and hydroquinone (HQ) produced, which indicates the formation of other reaction products such as polymeric phenols is almost none.

The amount of CTL and HQ was equal to phenol conversion, but reaction showed high selectivity for CTL as determined from the area under the GC chromatograms. The supported catalysts were recycled and also further evaluated for their catalytic activity after their applications in oxidation reactions. The efficiency of supported catalysts remained almost constant up to six recycles and after that the efficiency decreased (table 3) which may be due to either decomposition of the catalyst in the reaction media or their extraction to the organic solvent during product isolation. ${ }^{23}$ The product selectivity for CTL remained unaffected on using recycled catalysts, which was an indication for the structural stability of polymer supported metal complexes as confirmed by comparing IR spectra of recycled catalysts with IR spectra of freshly prepared catalysts. The activity of metal complexes in substrate conversion and product selectivity was evaluated at different molar ratios of substrate to hydrogen peroxide and catalyst.

The rate of phenol conversion was higher $(2.33 \times$ $10^{-6}$ mole $\mathrm{dm}^{-3} \mathrm{~s}^{-1}$ ) in the presence of polymersupported 3-MOBdMBn Schiff base complexes of nickel(II) ion than its unsupported Schiff base analogue $\left(1.37 \times 10^{-6}\right.$ mole $\left.\mathrm{dm}^{-3} \mathrm{~s}^{-1}\right)$ (table 4). The turnover number (TON) for the oxidation of phenol was higher (15.82 $\mathrm{g} \mathrm{mol}^{-1} \mathrm{Ni} \mathrm{hr}^{-1}$ ) in the presence of polymer supported Schiff base complex in comparison to unsupported Schiff base complex $\left(9.33 \mathrm{~g} \mathrm{~mol}^{-1} \mathrm{Ni} \mathrm{hr}^{-1}\right)$ at a molar ratio of $1: 1: 1$ of $\mathrm{H}_{2} \mathrm{O}_{2}$ to phenol and catalyst (table 4). The rate of substrate conversion and TON in the oxidation of phenol varied significantly with molar ratio of hydrogen peroxide but product selectivity in both cases remained almost constant (table 4).

\subsection{Effect of the $\mathrm{H}_{2} \mathrm{O}_{2}$ concentration/phenol concentration/catalyst concentration on the oxidation of phenol}

The oxidation of phenol was studied with the variation of the molar ratio of $\mathrm{H}_{2} \mathrm{O}_{2}$ to phenol from 0.5 to 2.0 at a constant molarity of the substrate and catalyst $(0.05 \mathrm{M})$ in acetonitrile $(2.0 \mathrm{~mL})$. The reaction temperature was maintained at $70^{\circ} \mathrm{C}$. When the molar ratio of $\mathrm{H}_{2} \mathrm{O}_{2}$ was varied from 0.5 to 1.0 , the oxidation of phenol increased in the presence of supported 3-MOBdMBn Schiff base complex of nickel(II) (figure 4). However, when the molar ratio of $\mathrm{H}_{2} \mathrm{O}_{2}$ was further increased $(>1)$ in the reaction mixture, the oxidation of phenol showed a decreasing trend, and a similar trend was observed with unsupported 3-MOBdMBn-Ni Schiff base complex analogue. The decreasing trend in the conversion of phenol was due to the decrease in molar ratio of phenol and catalyst with respect to molar ratio of $\mathrm{H}_{2} \mathrm{O}_{2}$. 
Table 3. Efficiency of recycled supported catalysts (P-3-MOBdMBn-M) towards oxidation of phenol.

\begin{tabular}{lcccccc}
\hline & \multicolumn{5}{c}{ Recycle number } \\
\cline { 3 - 7 } Supported Nickel complexes & 0 & 2 & 4 & 6 & 8 \\
\hline \multirow{2}{*}{ P-3-MOBdMBn -Ni } & Conversion & 68.8 & 68.0 & 67.1 & 64.8 & 43.2 \\
& Selectivity & 95.1 & 95.0 & 95.3 & 91.6 & 89.5
\end{tabular}

[Phenol]:[Catalyst]: $\left[\mathrm{H}_{2} \mathrm{O}_{2}\right]=1: 1: 1(0.05 \mathrm{M}), \mathrm{Temp} .=70^{\circ} \mathrm{C} . \mathrm{CH}_{3} \mathrm{CN}=2.0 \mathrm{~mL}$.

Table 4. Oxidation of phenol by [3-MOBdMBn-Ni]: Phenol conversion, selectivity for catechol and kinetic parameters.

\begin{tabular}{lcccc}
\hline Complexes & Conversion (wt $\%)$ & Selectivity (wt $\%)$ & TON & $\mathrm{R}_{\mathrm{p}}$ \\
\hline 3-MOBdMBn -Ni & 41.1 & 94.2 & 9.33 & 1.37 \\
P-3-MOBdMBn -Ni & 68.8 & 95.1 & 15.82 & 2.33
\end{tabular}

$\left[\mathrm{H}_{2} \mathrm{O}_{2}\right] /[$ Phenol $] /[$ Catalyst $]=0.05 \mathrm{M}$, Time $=1440 \mathrm{~min}$, Temp. $=70^{\circ} \mathrm{C} \mathrm{CH}_{3} \mathrm{CN}=$ $2.0 \mathrm{~mL}$ TON in terms of $=\mathrm{g} \mathrm{mol}^{-1} \mathrm{M} \mathrm{hr}^{-1}$, Rate of oxidation $\left(\mathrm{R}_{\mathrm{p}}\right)$ in terms of $=$ $10^{-6}$ mole $\mathrm{dm}^{-3} \mathrm{~s}^{-1}$

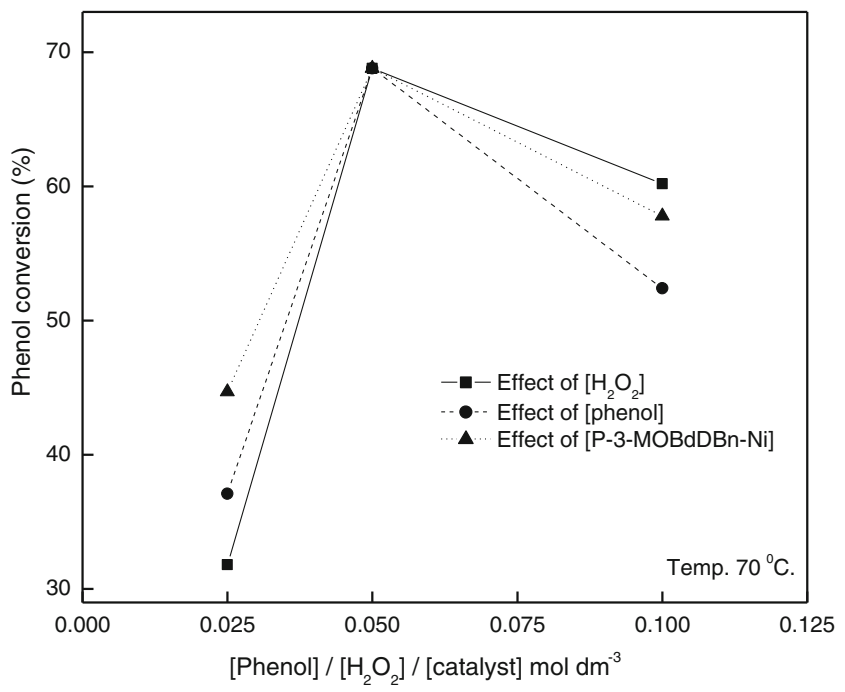

Figure 4. Effect of $\mathrm{H}_{2} \mathrm{O}_{2}$ concentration / phenol concentration / concentration of polymer-supported nickel complex catalyst towards oxidation of phenol.

The catalytic efficiency of the [3-MOBdMBn-Ni] complexes towards the oxidation of phenol was evaluated at different molar ratios of phenol in the reaction mixture, whereas the molar ratio of $\mathrm{H}_{2} \mathrm{O}_{2}$ to the catalyst was kept constant. The molar ratio of phenol was varied from 0.5 to 2.0 with respect to the molar ratio of $\mathrm{H}_{2} \mathrm{O}_{2}$ to the catalyst. During the molar ratio variation of phenol, the concentration of $\mathrm{H}_{2} \mathrm{O}_{2}$ and the catalyst was kept constant $(0.05 \mathrm{M})$. When the molar ratio of phenol was increased from 0.5 to 1.0 in the reaction mixture, the conversion (\%) of phenol increased substantially with polymer supported nickel complex of the Schiff base (figure 4), but when the molar ratio of phenol increased further $(>1)$, the conversion $(\%)$ of phenol showed a decreasing trend, which was due to the significant decrease in the molar ratio of $\mathrm{H}_{2} \mathrm{O}_{2}$ to the catalyst in the reaction mixture in comparison with the molar ratio of phenol. The oxidation of phenol was also evaluated at different molar ratios of polymer-supported 3-MOBdMBn Schiff base complex of nickel at a constant molar ratio (1: 1) of the substrate and oxidant. The molar ratio of Schiff base complex of nickel(II) ions was varied from 0.5 to 2.0 at a constant molarity $(0.05 \mathrm{M})$ of phenol and $\mathrm{H}_{2} \mathrm{O}_{2}$ in the reaction mixture. The conversion (\%) of phenol also showed the same trend as variation of substrate concentration.

\subsection{Mechanism for oxidation of phenol}

Considering the experimental findings for the oxidation of phenol with free and supported 3-MOBdMBn Schiff base complex of nickel ion, the following reaction steps are proposed (scheme 8). The free and polymer-supported Schiff base complexes of nickel ion (3-MOBdMBn-Ni) have produced active species (Ni3-MOBdMBn-HOO-) through fast interactions with $\mathrm{H}_{2} \mathrm{O}_{2}$ and 3-MOBdMBn Schiff base. The intermediate (Ni3-MOBdMBn-Ph-HOO-) has facilitated the nucleophilic attack of ${ }^{-} \mathrm{OOH}$ species on ortho and para position of phenol to produce hydroxy-substituted phenols (scheme 7). The reaction products, catalyst and hydroxyl ions were formed through decomposition of intermediates (scheme 8, step 4) and finally the 


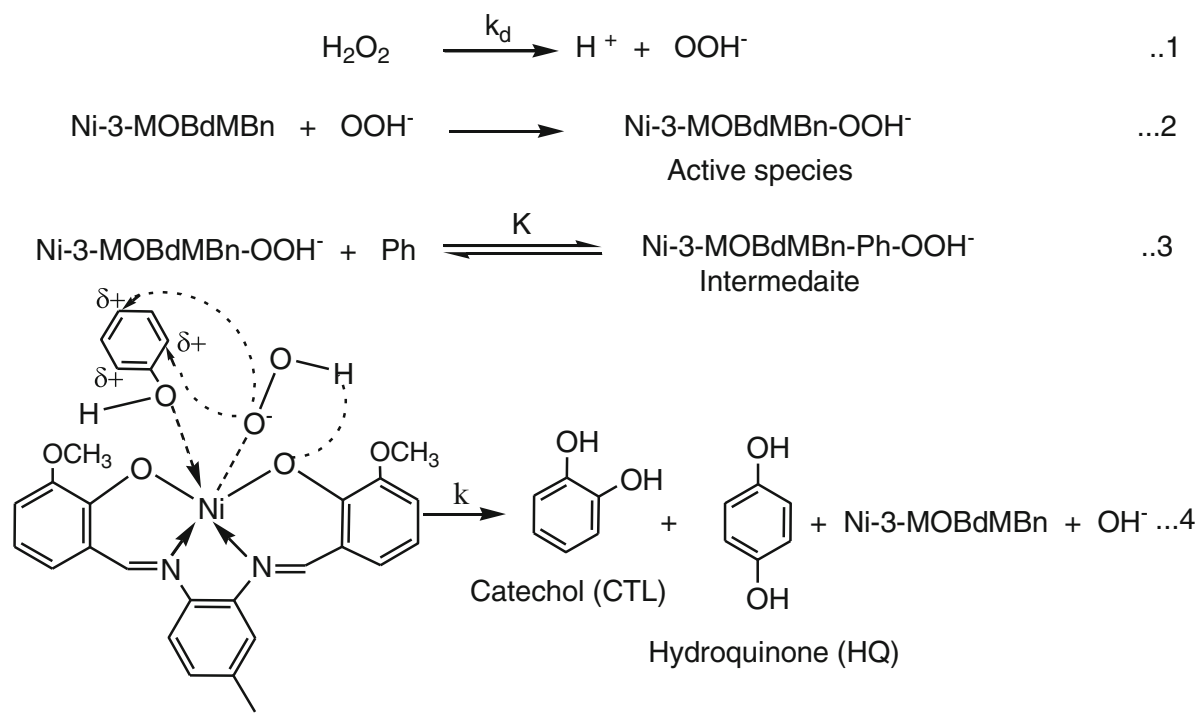

$\mathrm{Ni}-3-\mathrm{MOBdMBn}-\mathrm{Ph}-\mathrm{OOH}^{-}$

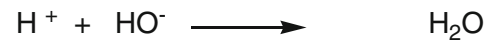

. .5

Scheme 8. Reaction steps of oxidation of phenol.

hydroxyl ions reacted with hydrogen ions, which were produced in step 1 .

\section{Conclusion}

The unsupported and polymer-supported nickel complexes of 3-MOBdMBn Schiff base were synthesized and characterized successfully for their structures and catalytic activity towards the oxidation of phenol. The polymer supported 3-MOBdMBn Schiff base nickel complex showed high catalytic activity than its free analogue. The oxidation of phenol showed high selectivity for catechol. The supported catalysts showed higher rate of oxidation and TON than unsupported catalysts, which clearly suggested that polymer support has played a significant role in increasing the rate for oxidation of phenol in the presence of nickel complexes of 3-MOBdMBn Schiff base. Concentration ration of $\mathrm{H}_{2} \mathrm{O}_{2}$ /phenol/catalyst on oxidation of phenol has a great effect in the presence of polymer-supported nickel complex. The oxidation of phenol was maximum at a molar ratio of $1: 1: 1$ of phenol to hydrogen peroxide and catalyst.

\section{Supporting Information}

FTIR spectrum of unsupported 3-MOBdDMBn Schiff base and polymer-supported 3-MOBdDBn Schiff base,
${ }^{1} \mathrm{H}-\mathrm{NMR}$ spectrum of 3 -MOBdMBn Schiff base, time variation data for conversion of phenol by unsupported and polymer-supported nickel complex (Ni-3-MOBdMBn) are provided in supporting information (SI) available at www.ias.ac.in/chemsci.

\section{Acknowledgements}

The authors are thankful to Department of Science and Technology (DST), Council of Scientific and Industrial Research (CSIR) and University Grants Commission (UGC), New Delhi, India for funding. The authors are also grateful to Ravenshaw University, KIIT University and National Institute of Technology, Raipur for providing research facilities.

\section{References}

1. Gupta K C and Sutar A K 2008 Coord. Chem. Rev. 252 1420

2. Chang Y, Zha F, Su B and Wang Y 2006 J. Macromol. Sci. Pure Appl. Chem. 43923

3. Ding K, Wang Z, Wang X, Liang Y and Wang X 2006 Chem. Eur. J. 125188

4. Thomas S R and Janda K D 2000 J. Am. Chem. Soc. 122 6929

5. Sutar A K, Maharana T, Dutta S, Chen C-T and Lin C-C 2010 Chem. Soc. Rev. 391724

6. Vatankhah-Varnoosfaderani M, Pourmahdian S and Afshar-Taromi F 2011 Iran. Poly. J. 20(11) 897 
7. Grivani G and Akherati A 2013 Inorg. Chem. Comm. 28 90

8. Gupta K C, Sutar A K and Lin C C 2009 Coord. Chem. Rev. 2531926

9. Yoo D W, Han J H, Nam S H, Kim H J, Kim C and Lee J K 2006 Inorg. Chem. Commun. 9654

10. Yue C, Fei Z, Bitao S and Yupu W 2006 J. Macromol. Sci. Part A Pure Appl. Chem. 43 (6) 923

11. Kumar A and Srinivas D $2013 \mathrm{~J} \mathrm{Mol} \mathrm{Catal} \mathrm{A:} \mathrm{Chem.}$ 368112

12. Keav S, de los Monteros A E, Barbier Jr. J and Duprez D 2014 Appl. Catal. B: Environ. 150402

13. Bellardita M, Augugliaro V, Loddo V, Megna B, Palmisano G, Palmisano L and Puma M A 2012 Appl. Catal. A: Gen. 44179

14. Hernmert C, Renz M and Meunier B 1999 J. Mol. Catal. A. Chem. 137205
15. Walling C 1975 Acc. Chem. Res. 8125

16. Gupta K C and Sutar A K 2008 J. Mol. Catal. A Chem. 280173

17. Owsik I, Kolarz B N and Jezierska J 2006 J. Catal. Lett. 107197

18. Gupta K C and Sutar A K 2007 J. Mol. Catal. A Chem. 27264

19. Gupta K C and Sutar A K 2007 J. Macromol. Sci. Part A: Pure Appl. Chem. 441171

20. Vogel A I 1978 In Textbook of practical organic chemistry (London: ELBS and Longman)

21. Fraile J M, Mayoral J A, Royo A J, Salvador R V, Altava B, Luis S V, Burguete M I 1996 Tetrahedron 52(29) 9853

22. Kowalak S, Weiss R C, Balkus K J 1991 J. Chem. Soc. Chem. Commun. 5757

23. Buijsman R C, van Vuuren E and Sterrenburg J G 2001 Org. Lett. 3(23) 3785 